\title{
Calicovesicostomy for ureteropelvic junction obstruction in a solitary ectopic pelvic kidney
}

Chavda S.K., M MED, FCS (ECSA), FICS, Nayyar R., MS, MCh, Singh P., MS, Gupta N.P., MS, MCh, FAMS Address for Correspondence: Dr. Narmada P Gupta MS, MCh, FAMS, All India Institute of Medical Sciences, N.Delhi-110029, India, Tel. No. (Office) 91-11-26594884 and 26593249, Fax- 91-11-26588663, 26588641, E-mail: narmadagupta@hotmail.com

\section{Abstract}

A solitary dysmorphic ectopic kidney is an uncommon congenital abnormality. This anomaly may be associated with ureteropelvic junction obstruction causing hydronephrosis and parenchymal thinning. We report such a case with non-dependent posteriorly placed pelvis in a pelvic kidney and its management. The perioperative results were satisfactory. This seems to be the first such case report of a calicovesicostomy used as a salvage procedure for an ectopic solitary kidney.

\section{Introduction}

Calicovesicostomy for a horseshoe kidney and giant hydronephrosis has been described $(1,2)$. This procedure offers an alternative to pyeloplasty or pyelovesicostomy in cases of uretero-pelvic junction obstruction. The major issue is to provide drainage for long term preservation of renal function. This is particularly pertinent in patients with poorly functioning kidneys. Here we report a case of congenitally absent left kidney and ectopic right kidney with ureteropelvic junction obstruction salvaged with calicovesicostomy.

\section{Case report}

A 15 year old female was admitted with pain and lump in the right lower abdomen for a period of 7 months. Pain was recurrent, acute onset, sharp and non-radiating and was relieved by analgesia. On abdominal examination, there was a mildly tender cystic mass in the suprapubic and right iliac fossa regions. Routine investigations including renal function tests were normal and urine microcopy showed pyuria. Urine culture was sterile. Abdominal ultrasonography revealed absence of normal renal tissue in the renal fossae on either side and a cystic mass in the right pelvic region. An intravenous urogram confirmed absence of renal tissue in either of renal fosse (Figure 1). Prompt excretion of contrast was seen in the hydronephrotic kidney on the right side of pelvis. It was large and globular with loss of reniform shape and thinning of renal parenchyma. Inferiorly, it was abutting on the right wall of the urinary bladder which was displaced towards the left side. Ureters were not visualized. A CECT confirmed above findings with no separate renal tissue seen apart from the pelvic mass (Figure 1). A Renal Dynamic Scan showed a hydronephrotic ectopic kidney in central pelvis with delayed clearance. An MCU showed a normal bladder with no VUR and no post void residual urine.

Cystoscopy revealed absence of left hemi-trigone and left ureteric orifice. Right RGP showed UPJO with high insertion of ureter. A diagnosis of a right solitary ectopic kidney with UPJO and high insertion of ureter was confirmed.

The patient was placed supine with the right side slightly elevated. A midline incision was made and kidney approached extraperitoneally. The kidney was malrotated with the pelvis and ureter directed posteriorly and also in a non-dependant position. The parenchyma was thin and kidney was filled with purulent urine. There were significant inflammatory changes around the UPJ. A decision was then made to proceed with calicovesicostomy instead of pyeloplasty or pyelovesicostomy. The peritoneum was reflected from the dome of urinary bladder. A $3 \mathrm{~cm}$ elliptical calicostomy was made over lowermost dependant portion of the kidney and the kidney tissue excised. A $3 \mathrm{~cm}$ 


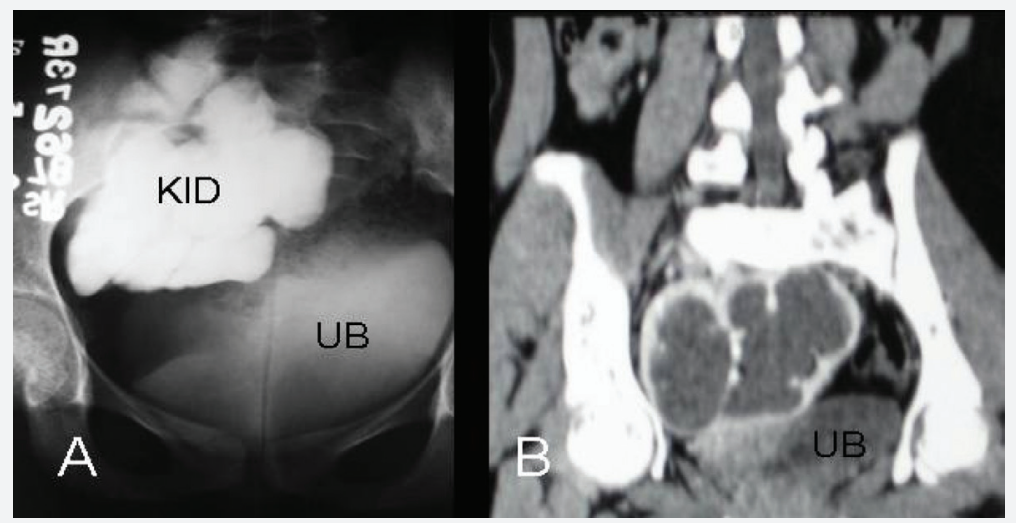

Figure 1. Pre-operative intravenous urogram (A) and CECT (B) showing an ectopic kidney (KID) with obstruction and faint opacification of the urinary bladder (UB). The Ureter was not visualized
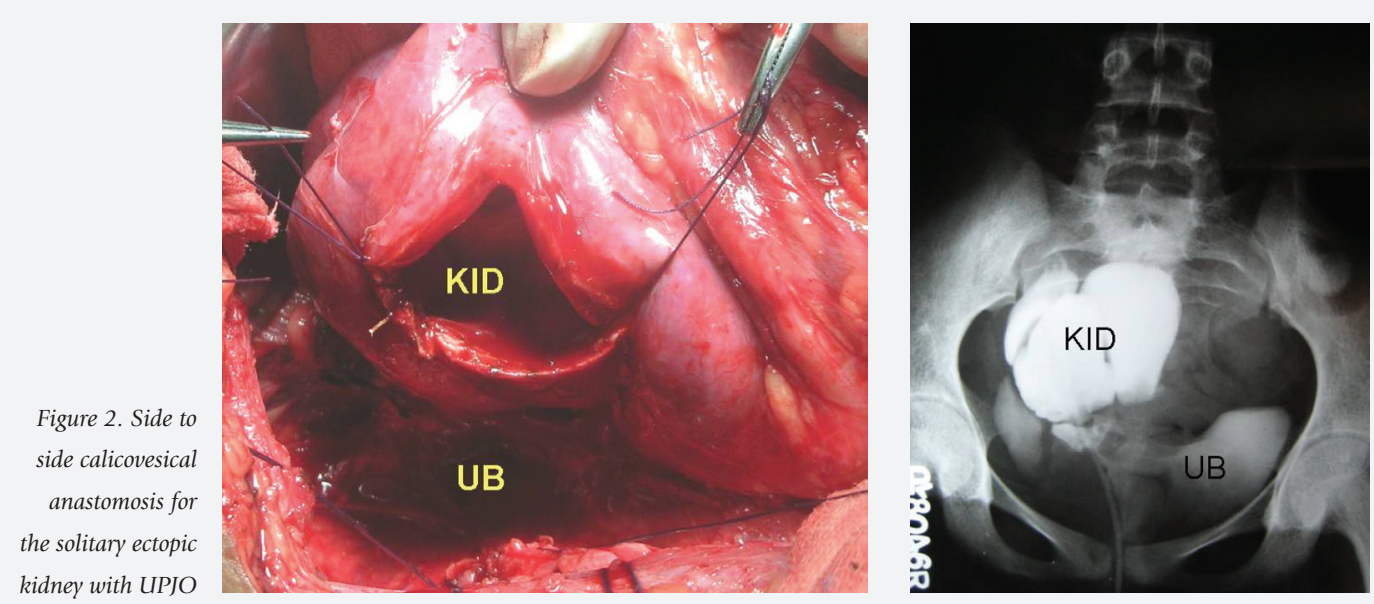

Figure 3. Post-operative cystogram depicting no leak around calycovesicostomy with a perurethral catheter in situ

cystotomy was made at the right bladder dome. Sideto-side calicovesical anastomosis was preferred in a continuous interlocking manner in a single layer using 3/0 polyglactin suture (Figure 2). A perurethral catheter was kept in the kidney with the balloon of the catheter resting above the anastomosis. Haemostasis was adequately achieved and wound was closed over a paravesical drain. The procedure was completed successfully in 97 minutes without any complications. The estimated blood loss was 140 mls. Post operatively, the patient was kept on antibiotics. She was allowed orally and resumed physical activity on 2nd post-operative day (POD). The drain was removed on the 3rd POD when she was discharged.

Post-operative cystogram done on POD 14 showed no evidence of contrast extravasation (Figure 3 ). The urethral catheter was then removed. At 6 months of follow up, the patient remains entirely asymptomatic with no UTI's and normal renal function.

\section{Discussion}

Urinary tract malformations account for $30 \%$ of all prenatally diagnosed congenital anomalies and are the predominant etiology for chronic renal failure in children (3). Ureteropelvic junction obstruction is associated with renal anomalies, and can occur in $22 \%$ to $37 \%$ of ectopic kidneys $(4,5)$. Little data is available in literature regarding the incidence of hydronephrosis in solitary, malrotated and pancake kidneys. In our case, the combination of solitary pelvic kidney with UPJO with hydronephrosis represents a rather uncommon urologic problem. The classic management options for UPJ obstruction associated with the ectopic kidney include open, laparoscopic or robotic pyeloplasty, open or laparoscopic partial nephrectomy, and endopyelotomy (retrograde or antegrade) $(6,7,8)$. However, these approaches may not provide the best clinical outcome in these patients. The other alternative to these different 
approaches is calicovesicostomy. The patient's large-capacity bladder provided an easier solution to this complex problem, because it was located in close proximity to the hydronephrotic lower pole of the right kidney. Without disturbing the natural course of right ureter and without dissecting all the neighboring renal vessels, a large calicostomy at the most dependent portion of the hydronephrotic right kidney was anastomosed to the large vesicostomy at the bladder dome. This approach minimized the potential risk of injury to the renal vasculature, pelvis and ureter.

The wider anastomosis made by calicovesicostomy would allow spontaneous passage of urine into the bladder and also make endoscopic manipulation easier, if required later. Further, factors like malrotation of the pelvicalyceal system and multiple aberrant arteries supplying a pelvic kidney, which could hamper performance of a satisfactory pyeloplasty, are circumvented by this procedure.

A drawback of this operation could be the possible detrimental effect of vesicocalyceal reflux upon renal function. In an experimental study using a canine model, it was possible to preserve excellent renal function for a year following vesicopyelostomy $(9,10)$. Similarly, these patients could develop renal back-pressure effects early if bladder outlet obstruction occurs at a later date, which may occur more commonly in males than females. Thus, these patients require a close life-long follow-up towards appropriate and timely intervention whenever a threat to renal function is detected.

Thus, calicovesicostomy to manage UPJ obstruction in a solitary pelvic kidney associated with gross hydronephrosis and parenchymal thinning is technically easier and was associated with satisfactory clinical outcomes.
After thorough medline search, this seems to be the first such case reported.

\section{References}

1 Mandal A.K., Hemal A.K., Vaidyanathan S: Boari flap calycovesicostomy: a salvage procedure for giant hydronephrosis due to ureteropelvic junction obstruction. J. Postgrad. Med. $1990 ; 36: 38-40$

2 Hsu T., Chang S., Hsu S.: Laparoscopic calicovesicostomy: A novel surgical procedure. Urology. 2006; 68: 413 - 415

3 Woolf A.S. A molecular and genetic view of human renal and urinary tract malformations, Kidney Int. 2000; 58: 500

4 Segura J.W., P.P. Kelalis and E.C. Burke. Horseshoe kidney in children, J Urol. 1972; 108: 333

5 P.E. Gleason, Kelalis P.P., Husmann D.A. et al. Hydronephrosis in renal ectopia: incidence, etiology and significance. J Urol. 1994; 151: 1660

6 Yohannes P. and Smith A.D. The endourological management of complications associated with horseshoe kidney, J Urol. 2002; 168: 5-8

7 Janetschek G., Peschel R. and Altarac S. et al. Laparoscopic and retroperitoneoscopic repair of ureteropelvic junction obstruction. Urology. 1996; 47: 311-316

8 Hsu T.H. and Presti J.C. Anterior extraperitoneal approach to laparoscopic pyeloplasty in horseshoe kidney a novel technique, Urology. 2003; 62: 1114-1116

9 Danforth, D. N. Jr., Javadpour, N., Bergman, S. M. and Terrill, R.. Pressure effects of urinary reflux studied with renal autotransplantation and pyelocystostomy. Urology. 1980; 15: $17-22$

10 Rao, K. M. K., Vaidyanathan, S., Reddy, M. J., Rao, et. al. Vesicopyelostomy for ureteropelvic junction obstruction in a solitary functioning pelvic kidney. Ind. J. Surg. 1980; 42: 204-208 